\title{
COMENTARIOS SOBRE EL ARTÍCULO: “DEPRESIÓN DEL ADULTO MAYOR PERUANO Y VARIABLES SOCIODEMOGRÁFICAS ASOCIADAS: ANÁLISIS DE ENDES 2017"
}

\author{
COMMENTS ON THE ARTICLE: “DEPRESSION OF THE PERUVIAN ELDERLY ADULT AND ASSOCIATED \\ SOCIODEMOGRAPHICAL VARIABLES, ANALYSIS OF ENDES 2017"
}

Jaime Lama-Valdivia, ${ }^{1,2, b}$, Rodrigo Rodríguez-Del Pozo 2,a

\begin{abstract}
Sr. Editor
En el artículo publicado por Baldeón-Martínez et al ${ }^{(1)}$ sobre depresión en el adulto mayor, los autores estimaron una prevalencia de depresión de 14,2\% en una muestra probabilística de 4917 personas mayores de 60 años de nuestro país. Los factores asociados a este diagnóstico fueron el sexo femenino, carecer de instrucción, edad mayor de 75 años, vivir en zona rural y ser pobre. Dicho estudio se realizó en base a los datos de la Encuesta Demográfica y de Salud Familiar (ENDES) 2017, utilizando para el diagnóstico de depresión el instrumento Patient Health Questionnaire (PHQ-9). El estudio presenta algunas fortalezas como la amplia cobertura, la calidad del muestreo y la información detallada y actualizada sobre los encuestados, sobre todo en los aspectos socio demográficos.
\end{abstract}

Sin embargo, su análisis permite encontrar una serie de limitaciones y sesgos que los autores no comentan en la discusión, como son: la metodología y objetivo del estudio, la validez del instrumento empleado en la población geriátrica y la relevancia o redundancia de la investigación respecto a trabajos publicados anteriormente sobre el tema.

El objetivo específico de la ENDES no fue estimar la tasa de prevalencia de depresión en el adulto mayor peruano, siendo la información del artículo obtenida de una base de datos secundaria. Por otro lado, los autores mencionan como objetivo determinar las variables asociadas a la depresión geriátrica, utilizando la fórmula de tamaño muestral de frecuencias la empleada por la ENDES y no una de factores asociados, lo cual sería lo más apropiado y que permitiría una interpretación correcta de los Odds Ratio (OR) obtenidos.

El instrumento utilizado fue el PHQ-9 validado en el Perú por un comité de expertos en salud mental, pero como Calderón et al ${ }^{(2)}$, mencionan en su artículo del 2012, la validación no se había hecho en la población, donde podrían existir diferencias socio culturales entre comunidades de la costa, sierra y selva. Siendo el instrumento completado mediante el auto reporte, los autores no mencionan los criterios de exclusión de su estudio como podrían ser: deterioro cognitivo crónico, delirium, hipoacusia, disminución de la agudeza visual, trastorno del aparato locomotor o barrera lingüística, todas ellas condiciones frecuentes en el adulto mayor ${ }^{(3)}$.

Revisiones recientes sobre los instrumentos utilizados en el diagnóstico de la depresión en el adulto mayor, concuerdan que la Geriatric Depression Scale (GDS) o Escala de Yesavage y la Escala Center for Epidemiological Studies-Depression (CES-D) son los instrumentos más confiables para su uso, al tomar en cuenta aspectos específicos de la presentación de esta patología en este grupo de edad, como son el déficit de atención y de memoria, las somatizaciones, la ansiedad y el trastorno del sueño ${ }^{4}$. De hecho, en la discusión de su trabajo,

\footnotetext{
'Facultad de Medicina Humana "Manuel Huamán Guerrero", Universidad Ricardo Palma, Lima-Perú.

${ }^{2}$ Hospital Nacional Hipólito Unanue, Lima-Perú.

a Médico geriatra.

Profesor asociado.

Citar como: Jaime Lama-Valdivia, Rodrigo Rodríguez-Del Pozo. Comentarios sobre el artículo: "Depresión del adulto mayor peruano y variables sociodemográficas asociadas: análisis de endes 2017". Rev. Fac. Med. Hum. Enero 2020; 20(1):166-167. DOI 10.25176/RFMH.v20i1.2560
} 
los autores comparan sus hallazgos con los obtenidos en estudios que utilizaron estos instrumentos.

Finalmente, el estudio no muestra mayor relevancia y hasta es redundante respecto a lo publicado por Martina et al ${ }^{(5)}$, quienes examinan la depresión y factores asociados en la población peruana adulta mayor según la ENDES 2014-2015, encontrando una prevalencia de depresión de 14,3\% y asociación a factores como el sexo femenino, ser mayor de 75 años, la carencia de instrucción, el vivir sin pareja, vivir en área rural, la pobreza y la discapacidad física.

Consideramos que son necesarios más estudios poblacionales sobre el tema, que aborden la depresión en el adulto mayor como una enfermedad, con un enfoque no sólo social y demográfico, sino también médico. Una propuesta podría ser estudiar los grupos identificados como de alto riesgo, junto con una valoración geriátrica integral, lo cual permitiría espacios de intervención oportuna ${ }^{6}$.

\section{REFERENCIAS BIBLIOGRÁFICAS}

1. Paola Baldeón-Martínez, Consuelo Luna-Muñoz, Sandra MendozaCernaqué, Lucy E. Correa-López. Depresión del adulto mayor peruano variables sociodemográficas asociadas: análisis de endes 2017. Rev. Fac. Med. Hum. Octubre 2019; 19(4):47-52. DOI 10.25176/RFMH.v19i4.2339

2. Calderón M, Gálvez-Buccollini JA, Cueva G, Ordoñez C, Bromley C, Fiestas F. Validación de la versión peruana del PHQ-9 para el diagnóstico de depresión. Rev Peru Med Exp Salud Publica [Internet]. diciembre de 2012 [citado el 1 de septiembrede2019];29(4):5789.Disponibleen:http:// www.scielosp.org/scielo.php?script $=$ sci_arttext\&pid=S1726 46342012000400027\&lng=es\&nrm=iso\&tlng=es

3. Varela L, Chávez H, Herrera A, Méndez F, Gálvez M. Perfil del adulto mayor INTRA II. Lima: Ministerio de Salud; 2004. 133p.
Contribuciones de autoría: Los autores participaron en la generación, recolección de información, redacción y versión final del artículo original.

Financiamiento: Autofinanciado.

Conflicto de interés: Los autores declaran no tener conflictos de interés en la publicación de este artículo.

Recibido: 15 de noviembre del 2019

Aprobado: 26 de diciembre del 2019

Correspondencia: Jaime Lama Valdivia.

Dirección: Av. Alfredo Benavides 5440, Santiago de Surco, Lima-Perú.

Teléfono: 999093383

Correo:jlamav@hotmail.com

4. Watson LC, Pignone MP. Screening accuracy for late-life depression in primary care: a systematic review. J Fam Pract. 2003; 52(12): 956-64.

5. Martina M, Ara MA, Gutiérrez C, Nolberto V, Piscoya J. Depresión y factores asociados en la población peruana adulta mayor según la ENDES 2014-2015. An la Fac Med [Internet]. el 23 de marzo de 2018 [citado el 1 de septiembre de 2019];78(4):393.Disponibleen:http:revistasinvestigacion.unmsm.edu.pe/ index.php/anales/article/view/14259

6. Gálvez-Cano M, Chávez-Jimeno H, Aliaga-Diaz E. Utilidad de la valoración geriátrica integral en la evaluación de la salud del adulto mayor. Rev Peru Med Exp Salud Publica. 2016;33(2):321-7.

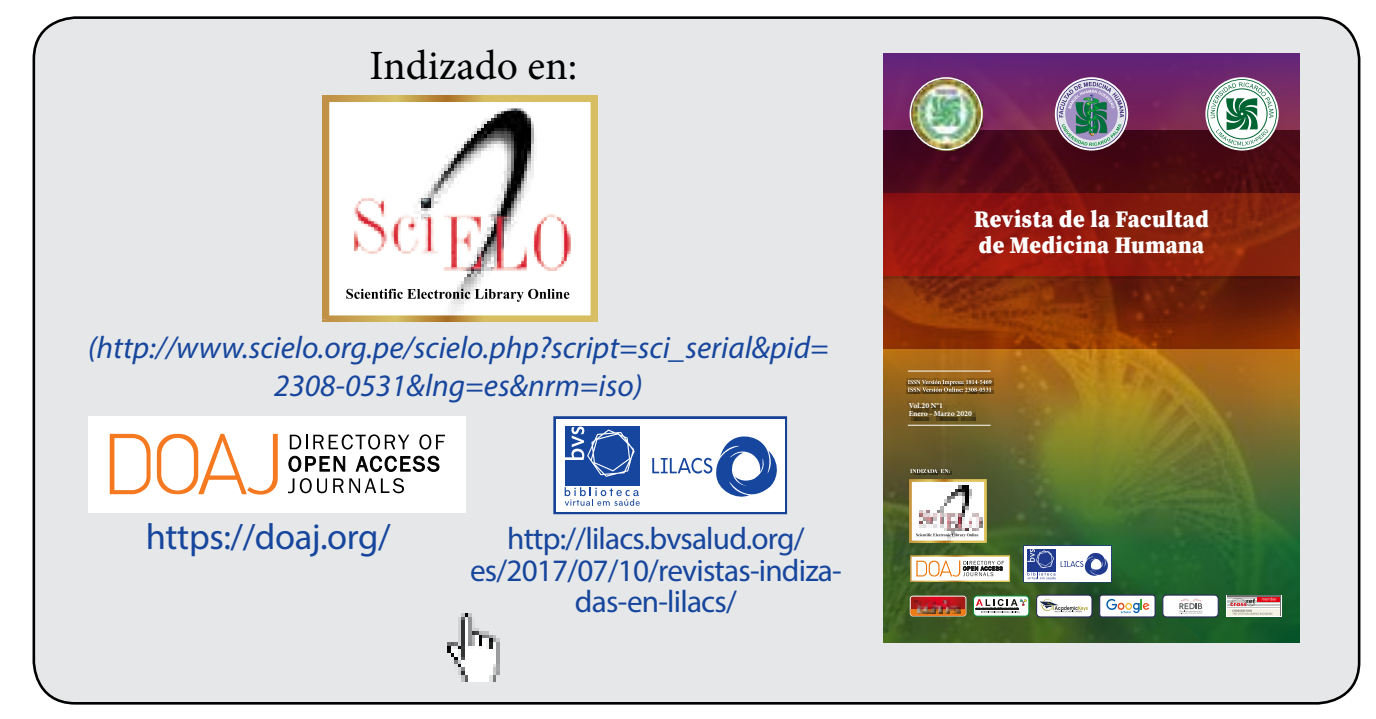

